\title{
Developing the Patient Falls Risk Report: A mixed methods study on sharing falls-related clinical information from home care with primary care providers
}

\section{Amanda Aguilar da Nova ( $\square$ aanova@uwaterloo.ca )}

\section{Research}

Keywords: interRAI-HC, home care, information sharing, falls, usability, utility, primary care

Posted Date: October 6th, 2021

DOl: https://doi.org/10.21203/rs.3.rs-951329/v1

License: (c) (1) This work is licensed under a Creative Commons Attribution 4.0 International License. Read Full License 


\section{Abstract}

Background: The interRAl home care (interRAl-HC) instrument is valid, reliable, and capable of enhancing integration across health settings. However, its uptake has been met with criticism. If interRAI-HC information were shared with primary care providers in a useable, actionable, and context-appropriate manner, evidence suggests that its implementation could enhance care provision and integration between these providers. The objective of this study was to co-develop an information sharing tool with primary care providers for sharing clinical information from the interRAI-HC (named the Patient Falls Risk Report).

Methods: This mixed-methods study, conducted from December 2019 to May 2020, employed qualitative and quantitative methods to develop and test the usability of the Patient Falls Risk Report. After recruiting primary care providers via snowball and maximum variation sampling, we employed semi-structured interviews in-person and over the telephone. The interview transcripts were analyzed through iterative thematic analysis and informed development of the report. Next, online surveys based on the System Usability Scale instrument were completed by a voluntary response sample of primary care providers and residents and descriptively analyzed to test the usability of the report.

Results: Of the interview sample $(n=9)$, we found that most believed that the Patient Falls Risk Report could support patient care by sharing relevant and actionable falls-related information. However, criticisms were identified including insufficient detail, lack of clarity, and limited support for shared care planning. After incorporating participants' suggestions for improvement, a sample of primary care providers and primary care residents $(n=27)$ determined that the report had excellent usability with an overall usability score of $83.4(95 \% \mathrm{Cl}=78.7,88.2)$.

Conclusions: By emphasizing usability and utility, and prioritizing the needs of end-users, sustainable interRAI-HC interventions can be developed and implemented to support care planning in primary care.

\section{Contributions To The Literature}

- Our findings show that complex information from assessment tools used in home care can be synthesized and adapted for use by primary care providers.

- Aligning with the content, language, organization, and delivery preferences of primary care providers can improve the usefulness of information sharing tools for them.

- The complex problems of fragmentation and burnout do not affect all health providers equally, yet they are important limitations to consider when trying to implement information-sharing tools in primary care.

\section{Background}

Integration is an organizational strategy for connecting the health system, enhancing performance, and improving quality of care [1]. Important components of integration include communication in a 
standardized clinical language, interdisciplinary collaboration, integrated electronic information systems, and appropriate funding mechanisms [2]. For persons with chronic and complex health conditions, enhanced integration can contribute to better health outcomes, cost-effectiveness, and quality of care [3].

The real-world application of integrated approaches has been suboptimal. Despite a large body of literature on best-practices, only $24 \%$ of Canadian primary care providers communicate with home care providers about the needs and services of their patients [4]. Additionally, one American study found that $96.3 \%$ of home care providers felt that their inability to obtain outside clinical information about their patients was problematic and $72.6 \%$ said that with access to outside clinical information, they would need to make fewer referrals to emergency departments [5]. The fragmentation between home care and primary care may prevent health care providers from fully appreciating a patient's clinical complexity, and, as a result, limit their ability to provide optimal care. Fragmentation is also associated with delayed care provision, repeat hospitalization, duplicate assessment, and other leading causes of adverse events [68]. For these reasons and more, generating evidence on innovations for enhancing the integration of care was identified as a strategic priority in the Canadian Institute of Health Services and Policy Research Strategic Plan for 2021 to 2026 [9].

One opportunity for enhancing integration between home care and primary care may be reinventing how results from the interRAl home care (interRAI-HC) assessment are used. The interRAl-HC is a comprehensive clinical assessment instrument used in home care to support care provision and improve health care quality [10-12]. As an instrument that is part of a suite of instruments used across the health sector, the interRAI-HC can be used to establish a shared understanding of patient needs between care settings, support care planning and transitions, reduce assessment duplication, and support the provision of high-quality integrated care [11].

However, the interRAI-HC is not being used to its full potential. While interRAl instruments are used across most of the Canadian health sector, many primary care providers in Ontario are unfamiliar with the interRAI-HC or are unaware of the functionalities and information available within the tool to support care planning $[13,14]$. Additionally, insufficient attention has been paid to the usability of interRAl information in clinical contexts [15]. The most common criticisms of the interRAl-HC among clinicians who use it are its lengthy paper format, inconsistent methods of delivery, and a perceived disconnect between the assessment results and goals of care [14].

\section{The Patient Falls Risk Report}

The Patient Falls Risk Report is a one-page report that was originally designed by the researchers of this study with knowledge from preliminary research and the Behaviour Change Wheel theoretical framework $[13,16-18]$. The original report held structured falls-related information derived from the interRAI-HC, including previous falls, cognitive impairment, pain, foot problems, inappropriate medication use, and physical inactivity. It also listed recommended interventions from the American Geriatrics Society and British Geriatrics Society Clinical Practice Guidelines for Prevention of Falls in Older Persons [19]. Each of 
the concerns listed in the Patient Falls Risk Report are prevalent among home care clients [20], can be addressed in primary care settings, and often go undiscussed, undisclosed, undetected, or deprioritized [19, 21-29]. The original Patient Falls Risk Report is attached in an additional file [see Additional file 1].

Upon implementation, primary care providers would receive the report by fax via the Client Health and Related Information System (CHRIS), a web-based electronic decision support and document management system that allows for the automated exchange of records [30,31]. Recipients would then be expected to schedule an appointment with the patient to discuss their results, collect missing information, and develop a care plan. Since falls are highly preventable with timely screening and assessment, we believed that sharing the report with primary care providers in a useable, actionable, and context-appropriate manner could enhance falls-related care planning $[13,14,16,19]$. The purpose of this study was to develop and test the usability of the Patient Falls Risk Report for sharing clinical information from home care to primary care in partnership with primary care providers.

\section{Methods}

This two-part mixed methods study employed in-depth interviews and short surveys to inform development of the Patient Falls Risk Report. Using qualitative and qualitative methods to provide complimentary perspectives on the report was expected to strengthen the reliability of our findings (96). AN and GH were both involved in carrying out the methods of this study.

\section{Interviews}

The purpose of the interviews was to develop of the Patient Falls Risk Report using the feedback of practicing primary care providers. Research shows that interventions are more likely to achieve their intended outcomes when the contexts, needs, and preferences of end-users are considered [32].

\section{Sample}

We aimed to recruit around 10 self-identified, English-speaking primary care providers for interviews who were practicing as family doctors, general practitioners, or nurse practitioners. A sample size of 10 was considered appropriate because, according to Kushniruk and Patel, 10 participants is enough to identify up to $80 \%$ of surface level issues of usability [33]. Additionally, a sample size of up to 10 was considered attainable given recruitment challenges identified in previous studies [34]. As a clinician and leader in their field, GH can be considered an insider in the clinical sphere. Therefore, GH was better connected to key informants and led recruitment. We used snowball and maximum variation sampling methods and sought to attain maximum variation on clinical background and training. Specifically, we aimed to include at least one nurse practitioner, one rural provider, one provider not in an interprofessional team, and one provider in an interprofessional health team. There were no exclusion criteria and recruitment continued until the maximum variation aims were met and saturation was achieved.

\section{Data Collection Procedures}


From December 2019 to February 2020, AN performed one-on-one qualitative interviews with primary care providers in Ontario and Alberta, Canada. As an early career researcher and Canadian graduate student, AN can be considered an outsider to the clinical research context; AN understood the topic of study but was not assumed to understand the day-to-day activities of a primary care provider. Consequently, participants were primed to provide more explanation on topics that would have been familiar to an insider [35]. Data collection was guided by usability testing methods and a constructivist theoretical approach, which posits that knowledge is jointly constructed and exists relative to social, historical, and cultural context $[32,36]$.

Prior to interviews, AN shared a consent form with participants and informed them that their interviews were confidential, and they could withdraw from the study at any time. Once written consent was obtained, data was collected with semi-structured interviews over telephone or at the location of participants' choosing.

The first interview questions explored participant experiences with falls prevention. Next, AN provided participants with a copy of the Patient Falls Risk Report with mock data [see Additional file 1] and asked them to propose care planning options, if necessary. Participants were then asked to describe their thoughts and feelings about using the report, if they would use it in their practice, and whether they believed it would change what they normally do in a patient encounter. Following this, participants were asked about their preferences for design and delivery of the report, potential barriers to implementation, and medicolegal risk. Finally, participants' type and duration in practice was identified (family doctor, general practitioner, or nurse practitioner), and additional comments or questions were solicited. The interview schedule is attached in an additional file [see Additional file 2].

The interviews were audio-recorded by a fingerprint-locked smartphone and, following each interview, reflexive notes on researcher thoughts, insights, and assumptions were taken by AN to improve dependability of the research process [37]. Within two weeks following each interview, the data were deidentified, transcribed, and stored by AN on a password-locked computer.

\section{Data Analysis}

AN analyzed the transcripts with Nvivo 12 using iterative thematic analysis. Each iteration of analysis began with a combination of deductive and open coding. Specifically, a coding framework based on the behaviour change wheel, usability testing, and preliminary research guided but did not constrain coding $[13,17,18,32]$. AN then grouped useful codes into themes and reviewed and mapped each theme to ensure a relationship to the overarching research topic. At the end of each iteration, the findings were summarized, and the Patient Falls Risk Report was revised accordingly. While reflecting on their outsider and insider perspectives, AN and GH jointly made decisions about changing the report based on availability of items within the interRAI-HC, critique frequency, and relevance to falls prevention in primary care. Following the final analysis, AN linked the findings to direct quotes and created a onepage infographic. The infographic was shared with participants via email for member checking to enhance trustworthiness of the findings. 


\section{Surveys}

The purpose of the surveys was to ensure credibility of the qualitative findings by evaluating the usability of the revised Patient Falls Risk Report, attached in an additional file [see additional file 3].

\section{Sample}

Survey recruitment was conducted by $\mathrm{AN}, \mathrm{GH}$, and MA. We aimed to recruit at least 20 primary care providers or primary care residents using voluntary response sampling via newsletter, email, and Twitter. The minimum sample size of 20 was determined using the System Usability Scale Calculator and research on expected recruitment limitations $[34,38]$. In the end, we anticipated that this sample would allow for an acceptable margin of error of about 10 points with a $95 \%$ confidence interval [38].

\section{Data Collection Procedures}

Data collection for the surveys was led by AN and took place from March to May 2020. To evaluate the revised Patient Falls Risk Report, participants were invited five-minute anonymous surveys. When participants opened the link to the survey on the Qualtrics XM platform, the purpose and procedures of the study, a description of the Patient Falls Risk Report, researcher contact details, and an informed consent question was displayed. Consent could be withdrawn at any time prior to survey submission. Once consent was provided, participants were shown the revised Patient Falls Risk Report with mock data and asked to identify at least two care planning options. This action was believed to have improved response accuracy [39]. The survey asked participants to rate 10 statements about the usability of the Patient Falls Risk Report on a Likert scale of one to five (from strongly disagree to strongly agree). If uncertain on the best response, participants were told to select the middle of the scale [39]. The survey questions were derived from the System Usability Scale, a robust, reliable, and valid industry standard [38, 40]. The System Usability Scale is used to score the usability of products and services on a scale of 0 to 100, where 100 represents the best possible usability [40]. Following completion of the survey, participants were given the option to provide additional comments in an open-ended comment box. The survey data was stored by AN in an Excel file on a password locked laptop.

\section{Data Analysis}

To prepare the quantitative data, individual scores on the System Usability Scale were calculated for each survey by AN using Excel 2004. Next, AN generated a histogram, box-and-whisker, and probability plot using SAS University Edition to evaluate the distribution of the scores. AN also conducted a Shapiro-Wilk test to determine if the System Usability Scale was used appropriately [38]. Next, the range, maximum, minimum, median, and average System Usability Scale scores were determined, and the standard deviation and confidence intervals for the average System Usability Scale score was calculated. Finally, AN performed benchmarking of the average score with the System Usability Scale curved grading scale [41]. This valid and reliable scale compares the usability of an innovation to thousands of other innovations [41]. Our aim was to achieve a score of at least 70, as recommended by Bangor et al. [40]. 
Finally, responses to the care planning activity and comments were reported for descriptive purposes. They would be analyzed more thoroughly if quantitative analysis indicated a need to improve usability of the Patient Falls Risk Report. In this situation, comments would be analyzed with thematic analysis, similar to the interviews.

\section{Ethics Considerations}

This study was reviewed for ethics clearance through a university research ethics committee and conforms to the Tri-Council Policy Statement: Ethical Conduct for Research Involving Humans (TCPS2).

\section{Results}

\section{Interviews}

After analyzing nine interviews, which were 26 minutes in length on average, and employing two iterations of qualitative analysis, we concluded that saturation was achieved since no new information emerged from the data. Four out of nine participants were nurse practitioners, one had worked in a rural practice, most practiced or had previously practiced as part of an interprofessional health team, and several had worked in a practice without an interprofessional team. The sample had practiced primary care for 21.7 years on average. One participant was based in Alberta, Canada and the rest were in Ontario, Canada.

Two overarching themes were identified from the interviews. The first was 'Perspectives on enhancing utility of the Patient Falls Risk Report' which had the subthemes, "It would help me provide really good care"', 'It should provide more information', and "'It's just another paper to file"'. Utility is defined in this paper as the quality of having the right features to solve a user need [42]. The second theme was 'Perspectives on enhancing usability of the Patient Falls Risk Report' and had the subthemes "It's easy to read"', 'The information in report is "not entirely clear"', and "'Fax is fine"'. Usability is defined in this paper as the ability for users to learn, understand, and operate a tool or system [42].

\section{Theme 1: Perspectives on enhancing utility of the Patient Falls Risk Report}

\section{"It would help me provide really good care"}

All nine participants claimed that they would use the Patient Falls Risk Report in their practice and seven said that the tool would impact how they interact with patients. To illustrate, one nurse practitioner described that the Patient Falls Risk Report could facilitate their conversations with patients: "I would probably show [my patient] the assessment and say 'I'm really concerned about this for you... Let's work together to try and make some changes to... decrease your risk and improve your health"' (NP1). The perception of utility stemmed from several described strengths of the report: it offers novel information from the home environment, supports critical thinking in assessment, prompts providers to address key risk factors in an evidence-informed way, and reminds them of recommended interventions and community resources. Most participants indicated that they would welcome the Patient Falls Risk Report 
because, "it would help me provide really good care" (NP1). When asked about the impact of potential medicolegal risks of implementation, one physician explained "I don't feel that as a problem. I mean anytime we get anything, be it a laboratory report, a consultant report, a nursing report. You know, if you ignore what it says then [laughs] that's not good" (MD5).

\section{It should provide more information}

To enhance utility of the Patient Falls Risk Report, several participants requested that it be "more detailed" (MD1). In particular, to "tease out the serious falls" (MD4), some participants wanted more detail around fracture risk, injuries, circumstances of the fall (e.g., loss of consciousness), and the patient's ability to stand, sit, and walk around. Other general suggestions included addition of narrative notes from the home care providers and information on demographics, drug and alcohol abuse, relevant chronic diseases (e.g., heart failure), and the state of the home environment. There were also several suggestions made regarding the list of risk factors. Four participants expressed the need for "more investigation" (NP3) around cognitive impairment. In terms of foot problems, one physician wanted more detail because, "pain versus wounds versus deformities are very different things" (MD1). Similarly, there was a suggestion to list the classes of high-risk medications prescribed to the patient and to indicate the prescriber. Participants also wanted to know more about pain; specifically, "where's the pain? When does it happen? What makes it better? What makes it worse?" (MD1) "is their pain well managed?... how is it managed? [and] Does it manage through physiotherapy?" (NP3). Finally, some participants suggested changes to the list of recommendations, such as including a list of local services or health providers who could be referred to, key numerical figures (i.e., bone mineral density, orthostatic vitals thresholds), and the actions that home care had taken. To facilitate access to outside support in particular, a nurse practitioner suggested emphasizing an eReferral management platform, "because I think people forget that you can go onto [the platform] and actually find the falls programs in our area" (NP4). While all of the suggestions can be considered important, it was not possible to add all of the details that participants requested without "getting bogged down in detail" (MD4) and exceeding a one-page length. Changes made to utility of the report are listed in Table 1. 
Table 1: Changes made to the Patient Falls Risk Report for improving utility

\section{Changes in Utility}

Added number of medications and list of high-risk mediations to medications status

Added "refer to pharmacist" to "conduct a medication review" in recommendations list

Added "home exercise program" to "refer to community exercise program" in recommendations list

Added "consider foot examination" to "consider podiatry assessment" in recommendations list

Added balance items to risk factors list

Added areas of impairment under cognitive performance status

Added "for the complete assessment, contact your home care provider" under risk factors list

Added pain control item to pain status and changed "pain" to "pain control"

Added "ensure up to date BMD" to "review bone health" in recommendations list

Added box on ways to get more information on community care resources in your area

\section{Justification}

Identifying the number and class of medications facilitates the medication review and related care planning.

Suggesting this concrete action for providers to take directly prompts them to reach out to allied health care providers

Broadens the conversation around exercise as prevention

Opens opportunity for provider to investigate if the foot problem is a wound, defect, or deformity since the interRAI-HC does not include this information

To provide understanding around patient's ability to stand, sit, and walk around and "tease out the serious falls" (MD4)

While this may not eliminate the need for a comprehensive cognitive assessment, it provides more details on where the problems lie.

If the provider is seeking additional detail about the state of the home environment, drug and alcohol abuse etc. they can request the complete assessment. These details were not included in the report to prevent 'information overload'.

Providing more detail around the pain can helps in prioritization of concerns for care planning.

Suggesting this concrete action for providers to take directly prompts them to evaluate fracture risk.

Informs providers on how they can access relevant community resources that can support falls prevention efforts. 
However, despite improvements made to the original report, two participants stated that receiving it would not change what they normally do in a patient encounter. One or these individuals indicated that they already collect the information in the report with custom-built comprehensive instruments and claimed to already know about the risk factors that their patients face. Specifically, one physician working in an interdisciplinary practice expressed their preference for an internally developed case finding program. The other provider felt that information provided was insufficient to support their needs. Instead, this nurse practitioner emphasized that they needed more support in managing the health of patients with complex conditions. They explained:

I think one of the struggles is time management. Trying to have the time to put towards these patients... It's a great tool, but the bottom line is: what are the resources that [the report is] going to get for me?... It's just another paper to file... it's not helping me get any resources... I didn't need a tool to tell me there's a problem with this person. I just need some help to figure out how I'm going to take care of them." (NP3).

In the same vein, other participants seemed to agree that being overwhelmed by a heavy workload was an important concern: "If everybody gives me a report like this for cognitive impairment, for mental health things, and... I have a hundred reports and I can't do it, then l'd rather have zero reports" (MD1). Therefore, several participants called for enhanced shared care planning: "I think if it is more of a community responsibility... you don't feel completely responsible, because often times... it does come back on to you" (MD4). However, challenges with shared care were identified: "shared care planning and interdisciplinary care, collaborative care, means different things to different people. And I think we all think we're doing it, but we don't do it very well" (MD2). Suggestions for preferred interventions included an automatic community referral system or a report which identified a list of actions taken by home care providers.

\section{Theme 2: Perspectives on enhancing usability of the Patient Falls Risk Report}

"It's easy to read"

Overall, the usability of the report was evaluated positively: "I like how it's laid out... I could look at this report in less than a minute and find out whether I need to act on it" (MD1). Characteristics reported to increase usability and make the report "easy to read" (NP1, NP2, NP4, MD2) included its one-page length, intuitive organization, simplicity of language and content, selection and emphasis of a limited number of key risk factors, and action items.

\section{The information in the report is "not entirely clear"}

Several critiques on the usability of the report itself were also identified. The few participants who commented on the falls overview section of the report sought clarification, but quickly found the answers to their questions within the report:

So, did my patient [pause] have a fall? I'm assuming they probably did- 'high risk is based on report of multiple falls'- So, then I'm assuming my patient did have a fall at least- I guess more than one. I guess that's not entirely clear maybe with the statements below. (MD3) 
Additionally, one participant explained that they would perform their own cognitive assessment based on the mock data, despite receiving the results of a valid cognitive assessment within the Patient Falls Risk Report, because: "I didn't get that they did a full cognitive assessment, because I don't know what they did to get that answer" (NP3).

The most prominent issue of usability that could be improved upon was lack of clarity around jargon in the report. In the first round of interviews, one participant expressed confusion around the interRAI jargon 'moderately impaired 1' under the section on cognitive performance. In fact, several participants indicated confusion with the numbers on the report:

I guess moderately impaired would mean something, but the 1 beside it means absolutely nothing to me and wouldn't to most primary care providers... most primary care people do not see RAI stuff at all... Is higher score worse or better?... That might want some clarification in case people needed to know (MD3).

The word 'triggered' within the medications and physical activities sections faced the same critique. Also in this section, the term "Inappropriate Medications" was labelled a "judgemental term" (MD1) since it implies blame on the prescriber and ignores contextual factors which may make the medication appropriate. Additionally, the meaning of the physical activities section in terms of lifestyle, physical condition, and motivations were unclear. Due to this lack of usability, the usefulness of the Physical Activities section was doubted by several participants. One participant in particular indicated that having conversations about exercise preferences are essential to developing an understanding of the item. To account for each of these critiques without increasing length, the information was reworded and rearranged. The changes made based on these critiques can be found in Table 2. 
Table 2: Changes made to the Patient Falls Risk Report for improving usability

\section{Changes in Reasoning \\ Usability}

Removed numerical scores

under

interpretations
While the numerical scores conveyed important information and may have increased utility of the report for those who understand them, they also decreased usability and created confusion for those who did not. Rather than adding a second page with interpretations of each score, which may also lower usability, it was agreed that the written descriptions were sufficient to support care planning.

Removed the ' 1 ' in 'moderately impaired 1' under cognitive performance status

Changed 'foot problems, no limitation in walking' to 'foot problems causing no limitation in walking'

Changed 'triggered' to 'major risk factor' or 'opportunity'

Moved medications section to the top of contributors to falls

Changed 'inappropriate medications' to 'medications' in medications section

Condensed reworded the falls overview to emphasize validity and meaning of the measure

Changed "physical activity" to "physical inactivity";
The ' 1 ' has little meaning to clinicians who are unfamiliar with interRAl-HC jargon.
Minor grammatical change
'Triggered' has little meaning to clinicians who are unfamiliar with interRAl-HC jargon.
Align report with what was most discussed by participants

Changing interRAI-HC jargon considered a "judgemental term" (MD1) without changing the meaning of the title

While saving space, this change also enhances usability by summarizing the assessment findings, interpretations, and high-level recommendations in one place. The section became more similar to an executive summary

These changes were made to convey how this item was measured and what it means about the patient in a concise and specific manner 


\section{"Fax is fine"}

Another key issue of usability was delivery of the Patient Falls Risk Report. While fax was described as a "fine" means of delivery by four participants (NP1, NP2, NP4, MD3), electronic medical record integration of the report "would be helpful" (MD5) according to those with the systems that allowed for it. One physician with decades of primary care experience summarized their views on the matter:

There's lots of people want to eliminate the fax. But I think the reality is it's pretty much in common use. I like it... The fax machine I think works for quite a few physicians still... I'm not the best person to ask because I depend on faxes. I still continue to get most of my messaging from other providers by fax. I have a process in place, but I think this is how people feel: that the fax machine is out of date, and they would rather there was electronic messaging. So, if I had a fully integrated [electronic medical record]. I may choose another method, but yeah, sorry (MD2).

While most would need to manually scan faxes into their systems, a task requiring time and effort, some participants reported using the Health Report Manager, which automatically uploads faxes into their electronic medical record: "through health report manager it actually comes in electronically. But fax is fine." (MD3). In summary, for most participants, integration with electronic medical records was preferred due to easier incorporation of the tool into their workflows.

\section{Surveys}

The sample size achieved for quantitative evaluation of the revised Patient Falls Risk Report (Fig. 2) was 27 primary care providers or primary care residents. The data from these participants was approximately normally distributed $(W$-Statistic $=0.94)$; therefore, use of the System Usability Scale was considered appropriate [38].

The overall System Usability Scale score for the revised Patient Falls Risk Report was 83.4 (SD=11.99) which is considered excellent on the System Usability Scale Benchmarking Scale at the $90^{\text {th }}$ to $95^{\text {th }}$ percentile [41] (Table 3). Additionally, the $95 \%$ confidence interval was within the range of acceptable scores [41]. In other words, the survey determined that the report is highly usable. Additionally, all participants completed the optional step and suggested care planning options. The most popular interventions suggested by survey participants were medication reviews, pharmacy referral, and referral to an exercise or balance program. There were also nine comments on the survey. Seven were short positive evaluations (e.g., "Great report!"), two were questions about the report ("who would complete this?" and "were the recommendations lists at the bottom just general suggestions for everyone or were they specifically recommended for my patient situation?"), and one was a suggestion to present risk factors in a more concise way. 


\begin{tabular}{|ll|}
\hline \multicolumn{2}{|l|}{ Table 3: Descriptive statistics for System Usability Scale scores } \\
\hline Mean & 83.4 \\
\hline 95\% Confidence Interval & $78.7,88.2$ \\
\hline Standard Deviation (SD) & 11.99 \\
\hline Median & 82.5 \\
\hline Mode & 100.0 \\
\hline Max & 100.0 \\
\hline Min & 62.5 \\
\hline
\end{tabular}

\section{Discussion}

This research shows that the Patient Falls Risk Report has the potential to support primary care providers in identifying risk factors and care planning options for patients receiving home care. The report was also determined to be usable and easy to understand. However, the participants suggested that poor shared care planning should be a key consideration for the development and implementation of frailty-related

information sharing tools.

Using structured approaches to sharing information between home care and primary care may motivate 'good care' by enhancing informational continuity, which refers to the ability of clinicians use information about patient medical history, conditions, context, and values to provide appropriate care $[13,43]$. Using interRAI-HC information in clinical practice is proven to be beneficial in supporting high-quality health care provision [10-12]. Proven benefits are integral for innovation sustainability, according to Fleiszer et al [44]. However, in information sharing, balance is key. While many participants in this study wanted more detail to be displayed in the report or had the means to conduct their own comprehensive case finding programs, many participants also described facing significant time constraints, burdensome workloads, and lack of support with managing complexity. Most primary care providers face heavy workloads [45], and addressing the complex needs of home care patients requires a complex interplay of clinical judgement and analytical thinking [46]. Therefore, we adjusted the report such that only information perceived as relevant and actionable to primary care was provided [18]. Of course, this adjustment was subjective and limited to information available within the original interRAl-HC assessment.

While the communication of relevant information is a necessary component of integration, providing more responsibility without minimizing burden in other ways can lead to loss of motivation, dissatisfaction, or burnout in primary care providers [45]. Since the Patient Falls Risk Report provides information without offering direct support to address falls risk, some participants felt as though work would be 'dumped' on them if this report were implemented. This view is justifiable. Adding detail on the actions of home care providers would have been beneficial for providing a more holistic view of patients, reducing the number of repeated referrals, and showing that home care is addressing patient health concerns [47]. Additionally, burden could be reduced by enhancing team-based care between primary care and allied healthcare providers (rather than care that is dominated by one provider), defining clear and manageable scopes of responsibility, and addressing electronic medical record limitations [45]. 
The one-page length, intuitive organization, simplicity, and actionability of the Patient Falls Risk Report were key usability-related strengths described by participants. Through the interviews, we were able to address many of the key criticisms of the interRAI-HC in the Patient Falls Risk Report [14]. For example, we attached information on community resources to the assessment results to support improved care planning. Engaging in usability-related changes may support incorporation of the report into existing primary care processes and structures, and, in turn, enhance sustainability of the innovation [44]. Additionally, usability of the tool among participants was improved by decreasing 'interRAl jargon'. While the use of a common interRAl language is a key characteristic of the instruments [11], 'translating' the assessment results, made it easier for participants to understand the assessment results presented to them. In the end, we found that the revised report was highly useable. High scores on the System Usability Scale correlate with greater task success [40,48], and usability itself can lead to ease of learning, ease of use, and intuitiveness, thus saving users time and increasing satisfaction with a product [32]. We recommend that future researchers intending to develop sustainable interRAl-HC innovations seek the perspectives of a diverse group of potential end-users throughout the development process and on an ongoing basis as needs change.

As a final comment, fax delivery may limit the usability of the Patient Falls Risk Report. Electronic records are an effective, practicable, and acceptable means of delivery due to easier integration of information into primary care workflows and enhanced decision support capabilities for improving patient outcomes $[3,18,46,47]$. However, electronic medical records as a delivery mechanism may also be unaffordable and inequitable since information sharing is an expensive functionality, limited to few system vendors [49]. Ontario's primary care sector needs more standardized data collection and management before delivery of the Patient Falls Risk Report by electronic medical record becomes feasible [48]. Ongoing development of the tool in terms of delivery (as well as usability and general utility) will be key in ensuring that the report is a sustainable intervention [44].

Strengths of this study included method triangulation, end-user involvement, and overall trustworthiness. By mirroring how humans naturally collect information, the combination of qualitative and quantitative data offered a rich information that would not have been possible otherwise [50]. In contrast, there were some notable limitations. This research may have been susceptible to volunteer bias (e.g., social desirability bias) due to nonprobability sampling. Most of the participants in this study practiced in Ontario and were likely to be more interested in system integration than the general population of primary care providers [51]. Additionally, since most of the interview participants practiced in interprofessional health teams, this study may overestimate primary care providers' knowledge of and connectedness with community resources. As an attempt to mitigate the issues that arose from nonprobability sampling, we used maximum variation sampling and assurance of confidentiality or anonymity [52]. We believe that we were successful in mitigating social desirability bias since dissenting views were reported for both overarching themes.

Moreover, the analysis of interviews and reporting of this study were shaped by worldviews of the researchers involved $[35,53]$. To improve credibility, we provided thick descriptions of themes, used data 
triangulation, and employed member checking to ensure that participant views were represented accurately. Finally, this study was limited by small sample sizes. Recruitment was challenging throughout this study due to limited time, resources, and motivation among primary care providers to participate in research, exacerbated by the COVID-19 pandemic [54]. To try and mitigate this challenge, we used snowball sampling in interview recruitment and the survey inclusion criteria was broadened to include primary care residents. Further description on the strengths and limitations of this study are recorded in the 'Good Reporting of A Mixed Methods Study (GRAMMS) Checklist' attached in an additional file [see additional file 4].

\section{Conclusion}

This research suggests that the Patient Falls Risk Report is a useful way to convey information derived from interRAI-HC assessments. It has the capacity to support primary care providers in identifying risk factors and engaging in care planning for patients with clinical complexity. The report also has the capacity to be sustainable. However, further consideration of clinician workloads, supportive resources (i.e., technological or human resources), and team-based approaches to care is needed. We also learned that, with the appropriate systems in place, sharing high-quality standardized information does not require imposing a standardized format. Utility and usability can support primary care frailty management and should be prioritized to benefit older persons with complex needs.

\section{Declarations}

\section{Ethics approval and consent to participate}

All participants in this study provided informed, written consent prior to participation. Participants were informed that they could withdraw from the study at any time up until results were submitted for publication (for interviewed participants) or until submitting the anonymous survey.

This study was reviewed for ethics clearance through the University of Waterloo Research Ethics Committee (ORE\#41215).

\section{Consent for publication}

Not applicable.

\section{Availability of data and materials}

The datasets used and/or analysed during the current study are available from the corresponding author on reasonable request.

\section{Competing interests}

The authors declare that they have no competing interests. 


\section{Funding}

The authors have no sources of funding for the research reported to declare.

\section{Authors' contributions}

AN, GH, LG, and MA all contributed substantially to study conception and design. GH and AN played primary roles in data acquisition and data interpretation. Specifically, AN and GH both designed the interviews, $\mathrm{GH}$ led participant recruitment, $\mathrm{AN}$ conducted and transcribed interviews, and $\mathrm{AN}$ and $\mathrm{GH}$ analyzed and interpreted the data. AN, GH, LG, and MA all participated in drafting and revision of the article and provided final approval of the version which we are submitting. AN, GH, LG, and MA also agreed to be personally accountable for their own contributions and to ensure that questions related to the accuracy or integrity of any part of the work, even ones in which they were not personally involved, are appropriately investigated, resolved, and the resolution documented in the literature.

\section{Acknowledgements}

Not applicable.

\section{Authors' Information}

AN holds a Bachelor of Science in Health Studies and a Master of Science in Public Health and Health Systems at the University of Waterloo in Ontario. They are currently a doctoral student completing a joint degree in the School of Public Health and Health Systems at the University of Waterloo and the Doctoral School of Biomedical Sciences in the Faculty of Medicine at KU Leuven.

GH holds a Doctor of Medicine degree with training in internal medicine from the University of Toronto, a geriatric medicine specialization from McMaster University, a fellowship from the Royal College of Physicians and Surgeons of Canada, and a Master of Science Degree in Health Research Methodology at McMaster University. They are currently the Schlegel Research Chair for Geriatric Medicine at the Research Institute for Aging, an Associate Professor with the School of Public Health and Health Systems at the University of Waterloo, and an Assistant Clinical Professor of Medicine at McMaster University.

LG completed their PhD at McMaster University. They have expertise in clinical exercise physiology, clinical trials and knowledge translation, physical activity and aging, and osteoporosis and bone health. LG is presently the Schlegel Research Chair in Mobility and Aging.

MA holds a Doctor of Medicine degree at the Schulich School of Medicine and Dentistry and completed their specialization in Family Medicine at McMaster University. They have completed a Masters of Health Information Science degree from the University of Victoria. They are currently an Assistant Clinical Professor in Family Medicine at McMaster University and the Managing Director at the eHealth Centre of Excellence where they work to implement ehealth initiatives. 


\section{Abbreviations}

interRAI-HC

interRAI home care

\section{References}

1. Kodner DL. (2009) All together now: a conceptual exploration of integrated care. Healthc Q Tor Ont 13 Spec No:6-15.

2. Suter E, Oelke ND, Adair CE, Armitage GD. Ten Key Principles for Successful Health Systems Integration. Healthc Q Tor Ont. 2009;13:16-23.

3. Martínez-González NA, Berchtold P, Ullman K, Busato A, Egger M. Integrated care programmes for adults with chronic conditions: a meta-review. Int J Qual Health Care. 2014;26:561-70.

4. Doty MM, Tikkanen R, Shah A, Schneider EC. Primary Care Physicians' Role In Coordinating Medical And Health-Related Social Needs In Eleven Countries. Health Aff (Millwood). 2019;39:115-23.

5. Vaidya SR, Shapiro JS, Papa AV, Kuperman G, Ali N, Check T, Lipton M. Perceptions of Health Information Exchange in Home Healthcare. CIN. 2012;30:503-9.

6. Masotti P, McColl MA, Green M. Adverse events experienced by homecare patients: a scoping review of the literature. Int J Qual Health Care. 2010;22:115-25.

7. Porter J, Herring J, Levinton JL and C (2007) ClHI Survey: Avoidable Admissions and Repeat Admissions: What Do They Tell Us? In: Healthc. Q.

http://www.longwoods.com/content/18645/healthcare-quarterly/cihi-survey-avoidable-admissionsand-repeat-admissions-what-do-they-tell-us-. Accessed 8 Jan 2021.

8. Toscan J, Mairs K, Hinton S, Stolee P, InfoRehab Research Team. Integrated transitional care: patient, informal caregiver and health care provider perspectives on care transitions for older persons with hip fracture. Int J Integr Care. 2012;12:e13-3.

9. Canadian Institutes of Health Research. (2021) Institute of Health Services and Policy Research Strategic Plan 2021-2026. 29.

10. De Almeida Mello J, Hermans K, Van Audenhove C, Macq J, Declercq A. Evaluations of home care interventions for frail older persons using the interRAI Home Care instrument: a systematic review of the literature. J Am Med Dir Assoc. 2015;16:173.e1-10.

11. Gray LC, Berg K, Fries BE, Henrard J-C, Hirdes JP, Steel K, Morris JN. Sharing clinical information across care settings: the birth of an integrated assessment system. BMC Health Serv Res. 2009;9:71.

12. Landi F, Onder G, Tua E, Carrara B, Zuccalá G, Gambassi G, Carbonin P, Bernabei R, Silvernet-HC Study Group of Bergamo. (2001) Impact of a new assessment system, the MDS-HC, on function and hospitalization of homebound older people: a controlled clinical trial. J Am Geriatr Soc 49:1288-93.

13. Nova AA, Zarrin A, Heckman GAW. Physician Views on the Resident Assessment Instrument for Home Care Information Exchange. J Am Med Dir Assoc. 2020;21:428-9.e1. 
14. Stolee P, Steeves B, Manderson BL, Toscan JL, Glenny C, Berg K. Health Information Use in Home Care: Brainstorming Barriers, Facilitators, and Recommendations. Home Health Care Serv Q. 2010;29:37-53.

15. LUCAS KU, Leuven. 21/03/2019 - From interRAI to BelRAI. Leuven, Belgium.

16. Guthrie DM, Pitman R, Fletcher PC, Hirdes JP, Stolee P, Poss JW, Papaioannou A, Berg K, Ezekiel HJ. Data sharing between home care professionals: a feasibility study using the RAI Home Care instrument. BMC Geriatr. 2014;14:81.

17. Michie S, Atkins L, West R. The Behaviour Change Wheel: A guide to designing interventions. Great Britain: Silverback Publishing; 2014.

18. Nova AA, Zarrin A, Heckman GAW. Physician Views on a Computerized Decision Support System for Home Care Information Exchange. J Am Med Dir Assoc. 2020;21:426-8.

19. Panel on Prevention of Falls in Older Persons, American Geriatrics Society, British Geriatrics Society. Summary of the Updated American Geriatrics Society/British Geriatrics Society Clinical Practice Guideline for Prevention of Falls in Older Persons. J Am Geriatr Soc. 2011;59:148-57.

20. Canadian Institute for Health Information. (2018) Profile of Clients in Home Care, 2017-2018.

21. Inouye SK. The dilemma of delirium: clinical and research controversies regarding diagnosis and evaluation of delirium in hospitalized elderly medical patients. Am J Med. 1994;97:278-88.

22. Schofield P. The Assessment of Pain in Older People: UK National Guidelines. Age Ageing. 2018;47:i1-22.

23. Williams AE, Blake A, Cherry L, Alcacer-Pitarch B, Edwards CJ, Hopkinson N, Vital EMJ, Teh LS. Patients' experiences of lupus-related foot problems: a qualitative investigation. Lupus. 2017;26:1174-81.

24. Wilson O, Kirwan J, Dures E, Quest E, Hewlett S. The experience of foot problems and decisions to access foot care in patients with rheumatoid arthritis: a qualitative study. J Foot Ankle Res. 2017. https://doi.org/10.1186/s13047-017-0188-3.

25. O’Brien MW, Shields CA, Oh PI, Fowles JR. Health care provider confidence and exercise prescription practices of Exercise is Medicine Canada workshop attendees. Appl Physiol Nutr Metab. 2017;42:384-90.

26. AuYoung M, Linke SE, Pagoto S, Buman MP, Craft LL, Richardson CR, Hutber A, Marcus BH, Estabrooks P, Sheinfeld Gorin S. Integrating Physical Activity in Primary Care Practice. Am J Med. 2016;129:1022-9.

27. Mackenzie L, Mclntyre A. How do general practitioners (GPs) engage in falls prevention with older people? A pilot survey of GPs in NHS England suggests a gap in routine practice to address falls prevention. Front Public Health. 2019. https://doi.org/10.3389/fpubh.2019.00032.

28. Mueller CA, Klaassen-Mielke R, Penner E, Junius-Walker U, Hummers-Pradier E, Theile G. Disclosure of new health problems and intervention planning using a geriatric assessment in a primary care setting. Croat Med J. 2010;51:493-500. 
29. Howland J, Hackman H, Taylor A, O'Hara K, Liu J, Brusch J. Older adult fall prevention practices among primary care providers at accountable care organizations: A pilot study. PloS One. 2018;13:e0205279.

30. Ontario Association of Community Care Access Centres. (2016) Connecting Care: OACCAC's eHealth Assets. 1-7.

31. Health Shared Services Ontario. (2017) Meet CHRIS. https://hssontario.ca/News/Pages/MeetCHRIS.aspx.

32. Barnum CM. Usability Testing Essentials. Burlington: Ready, Set... Test! Elsevier; 2011.

33. Kushniruk AW, Patel VL. Cognitive and usability engineering methods for the evaluation of clinical information systems. J Biomed Inform. 2004;37:56-76.

34. Johnston S, Liddy C, Hogg W, Donskov M, Russell G, Gyorfi-Dyke E. Barriers and facilitators to recruitment of physicians and practices for primary care health services research at one centre. BMC Med Res Methodol. 2010;10:109.

35. Holmes A. Researcher Positionality-A Consideration of Its Influence and Place in Qualitative Research-A New Researcher Guide. Shanlax int j educ. 2020;8:1-10. https://doi.org/10.34293/education.v8i4.3232.

36. Guba E, Lincoln Y. Competing paradigms in qualitative research. Handbook of qualitative research. 1994;2:105.

37. Tobin GA, Begley CM. Methodological rigour within a qualitative framework. J Adv Nurs. 2004;48:388-96.

38. Sauro J. A Practical Guide to the System Usability Scale: Background, Benchmarks \& Best Practices. Denver: CreateSpace Independent Publishing Platform; 2011.

39. Brooke J. SUS - A quick and dirty usability scale. In: Usability Eval. Ind. London: Taylor and Francis; 1996. p. 7.

40. Bangor A, Kortum PT, Miller JT. An Empirical Evaluation of the System Usability Scale. Int J HumanComputer Interact. 2008;24:574-94.

41. Sauro J, Lewis JR. Quantifying the User Experience: Practical Statistics for User Research. 2nd ed. Amsterdam: Morgan Kaufmann; 2016.

42. Nielsen J, Usefulness, Utility U. 3 Goals of UX Design [Internet]. 2017 [cited 2019 Sep 10]. Available from: https://www.youtube.com/watch?v=VwgZtqTQzg8.

43. Haggerty JL, Reid RJ, Freeman GK, Starfield BH, Adair CE, McKendry R. Continuity of care: a multidisciplinary review. BMJ. 2003;327:1219-21.

44. Fleiszer AR, Semenic SE, Ritchie JA, Richer M-C, Denis J-L. The sustainability of healthcare innovations: a concept analysis. J Adv Nurs. 2015;71:1484-98.

45. Agarwal SD, Pabo E, Rozenblum R, Sherritt KM. Professional Dissonance and Burnout in Primary Care: A Qualitative Study. JAMA Intern Med. 2020;180:395-401.

46. Dhaliwal G, Detsky AS. The Evolution of the Master Diagnostician. JAMA. 2013;310:579-80. 
47. Heckman HL, Manderson B, McKinnon-Wilson J, Santi SM, Stolee P. Developing an integrated system of care for frail seniors. Healthc Manage Forum. 2013;26:200-8.

48. Kortum P, Peres SC. The Relationship Between System Effectiveness and Subjective Usability Scores Using the System Usability Scale. Int J Human-Computer Interact. 2014;30:575-84.

49. Canadian Institute for Health Information. (2013) Insights and Lessons Learned From the PHC VRS Prototype. 16.

50. Wisdom J, Cresswell JW. Mixed methods: Integrating quantitative and qualitative data collection and analysis while studying patient-centered medical home models. Rockville: Agency for Healthcare Research and Quality; 2013.

51. Sedgwick P. Questionnaire surveys: sources of bias. BMJ. 2013;347:f5265.

52. Salkind NJ. Encyclopedia of research design. Thousand Oaks: SAJE Publications; 2010.

53. Anderson C. Presenting and evaluating qualitative research. Am J Pharm Educ. 2010;74:7.

54. Heckman, Saari M, McArthur C, Wellens NIH, Hirdes JP. COVID-19 outbreak measures may indirectly lead to greater burden on hospitals. Can Med Assoc J. 2020;192:E384-4.

55. Carter N, Bryant-Lukosius D, DiCenso A, Blythe J, Neville AJ. The use of triangulation in qualitative research. Oncol Nurs Forum. 2014;41:545+.

56. Abendroth TW. (1991) End-user participation in the needs assessment for a clinical information system. Proc Annu Symp Comput Appl Med Care 233-237.

\section{Additional Files}

\begin{tabular}{|llll|}
\hline File Name & $\begin{array}{l}\text { File } \\
\text { format }\end{array}$ & Title of data & Description of file \\
\hline $\begin{array}{l}\text { Additional } \\
\text { File 1 }\end{array}$ & $\begin{array}{l}\text { Microsoft } \\
\text { Word } \\
\text { Document } \\
\text { (docx) }\end{array}$ & $\begin{array}{l}\text { Original Patient } \\
\text { Falls Risk Report }\end{array}$ & $\begin{array}{l}\text { The original Patient Falls Risk Report was developed } \\
\text { by the researchers prior to conducting this study. It } \\
\text { was presented to interview participants. }\end{array}$ \\
\begin{tabular}{|llll} 
Additional \\
File 2
\end{tabular} & $\begin{array}{l}\text { Microsoft } \\
\text { Word } \\
\text { Document } \\
\text { (docx) }\end{array}$ & $\begin{array}{l}\text { Interview } \\
\text { Schedule }\end{array}$ & $\begin{array}{l}\text { This interview schedule contains the questions and } \\
\text { prompts used to conduct qualitative interviews in } \\
\text { this study }\end{array}$ \\
\hline $\begin{array}{l}\text { Additional } \\
\text { File 3 }\end{array}$ & $\begin{array}{l}\text { Microsoft } \\
\text { Word } \\
\text { Document } \\
\text { (.docx) }\end{array}$ & $\begin{array}{l}\text { Revised Patient } \\
\text { Falls Risk Report }\end{array}$ & $\begin{array}{l}\text { The revised Patient Falls Risk Report was developed } \\
\text { by the researchers using the feedback of the } \\
\text { interview participants. It was presented to survey } \\
\text { participants. }\end{array}$ \\
\hline $\begin{array}{l}\text { Additional } \\
\text { File 4 }\end{array}$ & $\begin{array}{l}\text { Microsoft } \\
\text { Word } \\
\text { Document } \\
\text { (.docx) }\end{array}$ & $\begin{array}{l}\text { Good Reporting of } \\
\text { A Mixed Methods } \\
\text { Study (GRAMMS) } \\
\text { Checklist }\end{array}$ & $\begin{array}{l}\text { The GRAMMS reporting guideline was obtained } \\
\text { from the EQUATOR Network website and was used } \\
\text { as a checklist to evaluate this mixed methods health } \\
\text { services research. }\end{array}$ \\
\hline
\end{tabular}

\section{Supplementary Files}


This is a list of supplementary files associated with this preprint. Click to download.

- AdditionalFile1.docx

- AdditionalFile2.docx

- AdditionalFile4.docx

- AdditonalFile3.docx 